\title{
Reproductive Health Screening Disparities and Sexual Orientation in a Cohort Study of U.S. Adolescent and Young Adult Females
}

\section{Citation}

Charlton, Brittany M., Heather L. Corliss, Stacey A. Missmer, A. Lindsay Frazier, Margaret Rosario, Jessica A. Kahn, and S. Bryn Austin. 2011. "Reproductive Health Screening Disparities and Sexual Orientation in a Cohort Study of U.S. Adolescent and Young Adult Females." Journal of Adolescent Health 49 (5) (November): 505-510. doi:10.1016/j.jadohealth.2011.03.013.

\section{Published Version}

10.1016/j.jadohealth.2011.03.013

\section{Permanent link}

http://nrs.harvard.edu/urn-3:HUL.InstRepos:34361427

\section{Terms of Use}

This article was downloaded from Harvard University's DASH repository, and is made available under the terms and conditions applicable to Other Posted Material, as set forth at http:// nrs.harvard.edu/urn-3:HUL.InstRepos:dash.current.terms-of-use\#LAA

\section{Share Your Story}

The Harvard community has made this article openly available.

Please share how this access benefits you. Submit a story.

Accessibility 


\title{
Reproductive Health Screening Disparities and Sexual Orientation in a Cohort Study of U.S. Adolescent and Young Adult Females
}

\author{
Brittany M. Charlton ${ }^{a}$, Heather L. Corliss, Ph.D. ${ }^{b, c}$, Stacey A. Missmer, Sc.D. ${ }^{a, d, e}$, A. Lindsay \\ Frazier, M.D. ${ }^{a, d, f}$, Margaret Rosario, Ph.D.g, Jessica A. Kahn, Ph.D. ${ }^{\text {h,i }}$, and S. Bryn Austin, \\ Sc.D. b,c,d,j \\ aDepartment of Epidemiology, Harvard School of Public Health, Boston, MA \\ bDivision of Adolescent and Young Adult Medicine, Children's Hospital, Boston, MA \\ 'Department of Pediatrics, Harvard Medical School, Boston, MA \\ ${ }^{d}$ Channing Laboratory, Department of Medicine, Brigham and Women's Hospital and Harvard \\ Medical School, Boston, MA \\ eDepartment of Obstetrics, Gynecology, and Reproductive Biology, Brigham and Women's \\ Hospital and Harvard Medical School, Boston, MA \\ fDana-Farber Cancer Institute, Boston, MA \\ gCity University of New York, City College and Graduate Center, New York, NY \\ hDivision of Adolescent Medicine, Cincinnati Children's Hospital Medical Center, Cincinnati, $\mathrm{OH}$ \\ University of Cincinnati College of Medicine, Cincinnati, $\mathrm{OH}$ \\ iDepartment of Society, Human Development, and Health, Harvard School of Public Health, \\ Boston, MA
}

\section{Abstract}

Purpose-To examine sexual orientation group disparities in Pap and STI/HPV tests among adolescents and young adult females.

\begin{abstract}
Methods-Survey data from 4,224 adolescents and young adults aged 17-25 who responded to the 2005 wave questionnaire of the Growing Up Today Study were examined cross-sectionally with multivariable generalized estimating equations regression. We examined associations between sexual orientation and reproductive health care utilization as well as abnormal results with completely heterosexuals as the referent group, controlling for age, race/ethnicity, geographic region, and sexual history.
\end{abstract}

Results-After accounting for sociodemographics and sexual history, mostly heterosexual/ bisexual females had 30\% lower odds of having a Pap test within the last year and almost $40 \%$ higher odds of being diagnosed with an STI, as compared to the completely heterosexual group.

(C) 2011 Society for Adolescent Medicine. Published by Elsevier Inc. All rights reserved.

Address correspondence to: Brittany M. Charlton, Department of Epidemiology, Harvard School of Public Health, 677 Huntington Avenue, $9^{\text {th }}$ Floor, Boston, MA 02115. bcharlto@ hsph.harvard.edu.

Publisher's Disclaimer: This is a PDF file of an unedited manuscript that has been accepted for publication. As a service to our customers we are providing this early version of the manuscript. The manuscript will undergo copyediting, typesetting, and review of the resulting proof before it is published in its final citable form. Please note that during the production process errors may be discovered which could affect the content, and all legal disclaimers that apply to the journal pertain. 
Additionally, lesbians had very low odds of having a Pap test in life $(\mathrm{OR}=.13$, $\mathrm{p}$-value $=<.0001)$ and having a Pap test within the last year $(\mathrm{OR}=.25$, $\mathrm{p}$-value $=.0002)$, compared to completely heterosexuals.

Conclusions-Our study demonstrates that sexual minority adolescent and young adult women underutilize routine reproductive health screenings including Pap smears and STI tests. Providers and health educators should be aware of these disparities so that they can provide appropriate care to young women and their families and ensure that all young women receive reproductive health screening. Further research is needed to explore reasons sexual minority females are not accessing care as recommended since this may suggest opportunities to improve reproductive health screenings as well as broader health care access issues.

\section{Key Terms}

Homosexuality, Female; Bisexuality; Heterosexuality; Sexuality; Sexual Behavior; Healthcare Disparities; Papanicolaou Test; Sexually Transmitted Diseases; Papillomavirus Infections

\section{Background}

Reproductive health care during adolescence and young adulthood helps prevent physical and psychological morbidity and improves health quality throughout life.[1] Acquiring a sexually transmitted infection (STI) is associated with increased risk for numerous adverse health outcomes, including other STIs, [2] infertility,[3] cancer,[4] and premature death.[5] The Human Papillomavirus (HPV) is the most common STI in the U.S.[2] with $80 \%$ lifetime incidence;[6] it most often afflicts adolescents with 74\% of new infections occurring in 15-24-year-olds.[2] HPV also acts as the primary cause of cervical cancer[7] by leading to cervical precancers. These can be screened with tools like the Papanicolaou (Pap) test,[8] but this test is underutilized and misunderstood, particularly by young adults[9] and sexual minorities (i.e., individuals who identify as lesbian, gay, or bisexual or who report same-sex sexual contact or attractions).[10]

Due to a number of increased risk factors, it is important to study reproductive health behaviors of adolescent and young adults as well as sexual minorities. The primary risk factor for HPV infection is contact with male partners so it is noteworthy that females of all sexual orientations often report a history of male sexual contact. Studies show that among sexually active adolescents, $76 \%$ of lesbian, $96 \%$ of bisexual, and $98 \%$ of heterosexual females report male sexual contact.[11] Even for women who have had sexual contact only with other women, HPV transmission is possible because it requires only skin-to-skin contact.[12,13] Additionally, adolescent girls have higher HPV rates compared to adult women because they tend to have more sexual partners than adults.[2] As compared to heterosexuals, sexual minority youth and adults are screened with Pap tests less frequently, possibly increasing their risk for cervical cancer.[14] Sexual minorities also have higher cigarette smoking rates in adolescence and adulthood[15] which increases the risk that an HPV infection will progress to a cervical abnormality.[16] [17, 18]

The Institute of Medicine reports that more data on STIs, Pap tests, and cervical dysplasia among sexual minorities is needed to guide medical, governmental, and educational policies. [19] Therefore, we examined Pap, STI, and HPV screening use and abnormal results in adolescent and young adult females of varying sexual orientations using descriptive epidemiologic research. This kind of analysis has rarely been conducted in a national sample. We hypothesized that sexual minority adolescent and young adult females underutilize Pap and HPV tests and have higher lifetime prevalence of abnormal results as compared cross-sectionally to heterosexual peers. Additionally, we hypothesized that 
reproductive health risk factors (sex of sexual partners, initiation of sexual intercourse, age of coitarche, and number of sexual intercourse partners) would differ by sexual orientation and partially explain sexual orientation group disparities in Pap test utilization, abnormal Pap test results, and STI/HPV risk in adolescent and young adult females.

\section{Methods}

\section{Study Sample}

The Growing Up Today Study (GUTS) is an ongoing longitudinal cohort study of US adolescents and young adults established in 1996 to assess an array of health topics. Women from the Nurses' Health Study II[20] who indicated they had at least one child aged 9 to 14 years provided consent and contact information for more than 25,000 of their children. Mothers who provided such information marginally varied from those who did not in regards to smoking ( $8 \%$ vs. $10 \%$ ), age (37.7 vs. 37.8 ), and body mass index (BMI) $(25.3 \mathrm{~kg} /$ $\mathrm{m}^{2}$ vs. $\left.25.7 \mathrm{~kg} / \mathrm{m}^{2}\right)$. Questionnaires were mailed to the children, and 9,039 girls $(68 \%)$ as well as 7,843 (58\%) boys returned completed questionnaires, indicating their consent. Questionnaires are now mailed annually or biennially, and more detailed information on the initial recruitment is available elsewhere.[21] Detailed reproductive health and sexual risk behavior questions were included on the 2005 GUTS questionnaire, so our sample was restricted to female participants responding in that survey year. Due to collecting this data in 2005, we included only participants who reported sexual orientation and met the 2005 Pap test eligibility guidelines of $\geq 3$ years since coitarche or $\geq 21$ years old $(\mathrm{N}=4,224)$.

Additionally, we conducted sensitivity analyses including those participants who would be eligible by the current Pap eligibility guidelines. Due to the small number of cases, analyses did not include participants who were unsure $(\mathrm{N}=6)$ or missing $(\mathrm{N}=14)$ the sexual orientation response. Because clinical guidelines now recommend screening begin at age 21 regardless of sexual history,[22] we ran additional analyses restricted to participants who met current Pap testing eligibility guidelines of $\geq 21$ years old regardless of sexual history $(\mathrm{N}=3,426)$. This study was approved by the Brigham and Women's Hospital institutional review board.

\section{Measures}

Sexual orientation-Sexual orientation was assessed with an item adapted from the Minnesota Adolescent Health Survey[22, 23] asking about feelings of attractions: "Which of the following best describes your feelings? (1) completely heterosexual (attracted to persons of the opposite sex), (2) mostly heterosexual, (3) bisexual (equally attracted to men and women), (4) mostly homosexual, (5) completely homosexual (gay/lesbian, attracted to persons of the same sex), (6) not sure." The "mostly homosexual" and "completely homosexual" responses were combined to form a lesbian category in order to increase statistical power. Participants who did not respond after two initial mailings received a short-form version of the 2005 questionnaire, which included the sexual orientation item. Encouraging participants to respond through multiple mailings and questionnaire forms resulted in more complete data.

Sexual history-Sex of sexual contacts was assessed with an item reading: "During your life, the person(s) with whom you have had sexual contact is (are)..." Responses included "I have not had sexual contact with anyone," "Females," "Males," or "Female(s) and Male(s)." An indicator variable was used for missing data on sex of sexual contacts $(\mathrm{N}=26)$. The next question read: "Have you ever had sexual intercourse? (By sexual intercourse we mean vaginal or anal sex)." It is important to note that this question uses a definition of sexual intercourse that excludes oral sex and that excludes other types of vaginal or anal sexual contact if a participant does not define it as intercourse. Responses included "yes," "no," and 
"not sure" and if a participant indicated "yes" or "not sure" they were prompted to answer: "During your life, with how many people have you had sexual intercourse?" and "How old were you when you had sexual intercourse for the first time?" The respective means within each sexual orientation category were used for any individual who indicated having initiated sexual intercourse but who were missing age $(\mathrm{N}=371)$ and number of sexual intercourse partners $(\mathrm{N}=428)$. All of the above questions appeared on the short-form survey version.

Care utilization-Pap test use was assessed with the question: "Have you ever had a Pap test?" Responses included "yes," "no," and "not sure." If a participant indicated "yes" or "not sure" they were prompted to answer another question: "Have you had a Pap test in the past year?" with "yes," "no," and "not sure" possible responses. Physical exam use was also assessed with the question: "When was your last routine (preventative) physical exam or check-up?" The short-form survey version included both Pap test items but not the physical exam item. No information on HPV vaccine utilization was collected.

Abnormal results—Participants who indicated "yes" or "not sure" for "ever had a Pap test" were also prompted to respond to a third question: "Have you ever been told by a doctor or nurse that you had an abnormal Pap test?" with "yes," "no," and "not sure" possible responses. A separate question assessed STI diagnoses: "Have you ever been told by a doctor or nurse you have a sexually transmitted disease or STD? (for example: chlamydia, genital herpes, gonorrhea, genital warts, pubic lice or crabs, syphilis, HIV or AIDS)." Responses included "yes," "no," and "not sure." If a participant indicated "yes" or "not sure," they were prompted to answer a second question: "Have you ever had human papillomavirus (HPV) infection or genital warts?" with "yes," "no," and "not sure" possible responses. The short-form survey version did not include questions about abnormal results.

\section{Statistical Analysis}

We conducted descriptive statistics and multivariate regression analyses with SAS statistical software 9.2 (PROC GENMOD).[24] Analyses were cross-sectional and used 2005 data. We investigated sexual orientation group patterns in the prevalence of care utilization and abnormal results. We then examined multiple covariates that may confound or explain associations between sexual orientation and care utilization or abnormal result variables. First, we tested sociodemographic covariates (age, race/ethnicity, and region of residence) in separate models for each explanatory variable. Next, we tested sociodemographic and sexual history covariates (any male sexual contacts, initiated sexual intercourse, age at coitarche, and number of sexual intercourse partners). We used generalized estimating equation (GEE) statistical methods in regression analyses to account for intracluster correlation resulting from sibling groups in the cohort.[25] An indicator variable for missing data was created for race $(\mathrm{N}=18)$, while missing data on geographic region were not included in the analysis due to the small number of cases $(\mathrm{N}=7)$. Models estimated odds ratios $(\mathrm{OR})$ and $95 \%$ confidence intervals (CI) using completely heterosexual as the reference group. Mostly heterosexual and bisexual were collapsed to form one category in the GEE models because associations with predictors and outcomes were similar in the two groups and combining the groups increased statistical power.

\section{Results}

Among the 4,224 female participants included in our analyses, $82 \%(\mathrm{~N}=3,478)$ described themselves as completely heterosexual, $14 \%(\mathrm{~N}=572)$ as mostly heterosexual, $3 \%(\mathrm{~N}=114)$ as bisexual, and $1 \%(\mathrm{~N}=60)$ as mostly homosexual or completely homosexual (lesbian). Table 1 displays characteristics of the study population by sexual orientation. The racial/ ethnic composition of the cohort was predominantly white (93\%), and participants were 
aged 17 to 25 years when they returned the 2005 questionnaire with a mean of 21.6 years ( $\mathrm{SD} \pm 1.4)$. Overall, compared to completely heterosexuals, females who identified as mostly heterosexual, bisexual, or lesbian were more likely to report that they had initiated sexual intercourse, and mostly heterosexuals as well as bisexuals reported a younger age of coitarche and more sexual partners. A high proportion of participants, regardless of sexual orientation, reported some kind of male sexual contact: $63 \%$ of lesbian, $96 \%$ of bisexual, $96 \%$ of mostly heterosexual, and $89 \%$ of completely heterosexual females.

Table 2 displays prevalence estimates of STI and Pap tests by sexual orientation groups. Mostly heterosexual adolescents and young adults had the highest Pap test use while lesbians reported the lowest Pap test use within the last year. Among individuals who reported ever having a Pap test, the abnormal Pap tests rate was consistent across each sexual orientation group at around 20\%. Among individuals who reported an STI diagnosis, HPV diagnoses were also constant across each sexual orientation group at about $66 \%$. STI diagnoses were most common in the mostly heterosexual and bisexual groups. We also examined if the prevalence of a recent routine physical exam varied by sexual orientation. All of these associations were statistically significant with p-values <.0001. Compared to $59 \%$ of completely heterosexual females, $53 \%$ of mostly heterosexual (p-value $=<.0001$ ), $54 \%$ of bisexual (p-value $=.0078$ ), and $47 \%$ lesbian females ( $\mathrm{p}$-value $=.4178$ ) report a routine physical exam within the last year.

In order to examine associations between sexual orientation and the reproductive health outcomes before and after adjusting for sexual history, we restricted the remaining analyses to the subsample of females who reported on the sex of their sexual contacts, their age of coitarche, and the lifetime number of their sexual intercourse partners $(\mathrm{N}=3,590)$. Table 3 shows the partially-adjusted ORs from the models controlling for sociodemographic variables and then the fully-adjusted ORs from models further controlling for sexual history variables. After adjusting for age, race/ethnicity, and geographic region, reporting a mostly heterosexual/bisexual sexual orientation was positively associated with ever having a Pap test and an STI diagnosis but negatively associated with having a Pap test within the last year when compared to completely heterosexuals. Reporting a lesbian as compared to a completely heterosexual orientation was negatively associated with ever having a Pap test and having a Pap test within the last year. Most ORs that had been significant in partially adjusted models remained significant in the fully adjusted models, though some were attenuated. After accounting for sociodemographics and sexual history, mostly heterosexual/ bisexual females had 30\% lower odds of having a Pap test within the last year and almost $40 \%$ higher odds of being diagnosed with an STI, as compared to the completely heterosexual group. In fully adjusted models, lesbians had very low odds of having a Pap test in life $(\mathrm{OR}=.13$, $\mathrm{p}$-value $=<.0001)$ and having a Pap test within the last year $(\mathrm{OR}=.25$, $\mathrm{p}$ value $=.0002$ ), compared to completely heterosexuals.

Based on the new clinical guidelines for Pap screening released in late 2009, we ran subanalyses restricted to participants who met the new eligibility guidelines of $\geq 21$ years old regardless of sexual history $(\mathrm{N}=2,792)$. Adjusting for sociodemographics, mostly heterosexual/bisexual women still had higher odds of receiving an STI diagnosis (pvalue $=<.0001)$ and lower odds of lifetime Pap test ( $\mathrm{p}$-value $=.0017)$ while lesbian women still had lower odds of obtaining a Pap test within the last year ( $\mathrm{p}$-value $=<.0001)$ (data not shown).

\section{Discussion}

Our study demonstrates that sexual minority adolescent and young adult women underutilize routine reproductive health screenings including Pap smears and STI tests. Both lifetime and 
past-year Pap testing as well as history of STI diagnosis varied by sexual orientation. After accounting for sociodemographics and sexual history, mostly heterosexual/bisexual females were less likely than heterosexual females to have had a Pap test in the past year but more likely to have ever had an STI. Lesbians were far less likely to receive Pap test screening than completely heterosexuals, even when accounting for sociodemographics and sexual history.

Prior studies document that sexual minority youth do not have as much support from family and heterosexual peers around sexuality issues[26] and they do not believe they are at risk for abnormal Pap tests and STI diagnoses due to their sexual orientation,[27] which might lead to lower rates of sexual health screenings like Pap smears and STI tests. Less social support,[26] lower risk perceptions,[27] or an initial adverse experience like a clinician assuming a particular sexual orientation which is also linked to poorer reproductive health outcomes[13] could lead to infrequent care and higher abnormal Pap test and HPV diagnoses rates. All of these mechanisms should be explored in future research. Clinicians should understand these barriers and communicate to patients that certain sexual behaviors, regardless of sexual orientation identity, increase risk. Our finding that mostly heterosexual/ bisexual females had higher odds of being diagnosed with an STI, compared to completely heterosexuals, is consistent with previous findings in adults.[11, 31, 32] No sexual orientation differences were observed in the odds of receiving an HPV diagnosis, but there were few reported cases. HPV DNA is present in almost all invasive carcinomas and the vast majority of abnormal Pap test results.[28] However, more than double the number of participants who reported an HPV diagnosis reported an abnormal Pap test, suggesting that participants may have a poor understanding of the meaning and cause of an abnormal Pap test. The lower rates of even routine physical exams use in sexual minorities compared to completely heterosexuals underscore how important it is to encourage sexual minority adolescent girls and young women to regularly seek care.

Lesbian adolescents and young adults had lower odds of having a Pap smear, both over their lifetime and in the past year. Again, possible factors contributing to this disparity include a lower risk perception,[29] poor patient-provider communication,[10] lack of insurance,[13] or other health care system factors.[30] Regardless of a patient's reported sexual orientation identity, clinicians must offer a Pap test to every individual who meets age guidelines. Lesbians may be less likely to use birth control and therefore miss regular health care opportunities. Future studies with greater statistical power may reveal that lesbians have lower odds of abnormal Pap tests and STI diagnosis, but examining comparisons across sexual orientation groups will continue to be difficult even in large studies because of care underutilization by lesbians, since cervical dysplasia and most STIs are asymptomatic and will not be detected without regular preventative care. Previous studies report that lesbian adolescents[11] and adults[19, 31, 32] are at an increased risk for STIs but are unlikely to report receiving a diagnosis.[33]

Although our study had numerous strengths like the large, national sample, there were limitations. While we were able to examine a younger population compared to prior studies, we were limited by the homogenous racial/ethnic composition of our cohort and by the fact that all of the participants are children of nurses. Daughters of nurses may have higher health care utilization than the general population, which would overestimate utilization in this sample. Therefore, the sample is not representative of US adolescents and young adults. All of the data were available only from self-reports rather than another source such as medical records. One of the limitations of self-reports in this context is that any gynecological exam could be misreported as a Pap test, which could lead to over-reporting. Due to working with existing data, we could not access any other information such as more detailed sexual history variables. Additionally, our data likely underestimate the true 
prevalence of our outcomes because many abnormal results, like an asymptomatic STI, go undetected and previous research indicates that adolescent and young adult women do not reliably report detected diagnoses like HPV infections.[34] Restricting the analysis to 2005 Pap guidelines did not appear to bias the results when we reanalyzed the data using current guidelines in the sensitivity analyses. However, the new Pap test guidelines may impact reproductive health screenings among sexual minorities. Now that eligibility is based on age rather than a combination of age and sexual history, sexual minority woman may have more similar care to their heterosexual peers. More research is needed to explore these associations. Lastly, we had low power to detect differences in some outcomes such as abnormal Pap tests and HPV diagnoses. Another important strength of our study is that while previous research has varied the operational definition of "lesbian,"[10] often combining behavior and identity measures or even including bisexual women in this category, we were able to more precisely examine different sexual orientation identity groups by distinguishing between lesbian and bisexual women and controlling for sexual history.

Regular Pap tests and STI screenings are needed by all young adult females who meet current guidelines, regardless of sexual orientation. Despite changes in Pap test guidelines, STI screenings recommendations have remained consistent. The new Pap test recommendations, which suggest screening begin at age 21 regardless of sexual history, should make it easier for providers to determine those in need of screening. Even when we restricted our analysis to women aged 21 years and older, most of the sexual orientation group disparities remained. Providers and health educators should be aware of these disparities so that they can provide appropriate care to young women and their families and ensure that all young women receive reproductive health screening. Many adolescents and young adults encounter barriers to HPV and STI screening such as care site access problems (geography and hours), privacy concerns, and fear of disclosing stigmatized or risky sexual behavior.[30] Sexual minority youth may be further deterred especially in settings perceived as insensitive to their specific concerns. Further research is needed to explore reasons sexual minority females are not accessing care as recommended since this may suggest opportunities to improve reproductive health screenings as well as broader health care access issues.

\section{Acknowledgments}

This study was supported by research grant R01HD057368 from the National Institutes of Health. HL Corliss and SB Austin are supported by the Leadership Education in Adolescent Health Project grant T71 MC 00009-17 from the Maternal and Child Health Bureau. HL Corliss is also supported by the National Institute on Drug Abuse grant K01 DA023610.

\section{References}

1. Igra V, Millstein SG. Current status and approaches to improving preventive services for adolescents. JAMA. 1993; 269(11):1408-1412. [PubMed: 8441217]

2. Hager WD. Human papilloma virus infection and prevention in the adolescent population. J Pediatr Adolesc Gynecol. 2009; 22(4):197-204. [PubMed: 19481480]

3. Lindeque BG. Management of cervical premalignant lesions. Best Pract Res Clin Obstet Gynaecol. 2005; 19(4):545-561. [PubMed: 16150393]

4. zur Hausen H. Papillomaviruses in the causation of human cancers - a brief historical account. Virology. 2009; 384(2):260-265. [PubMed: 19135222]

5. Lizano M, Berumen J, Garcia-Carranca A. HPV-related carcinogenesis: basic concepts, viral types and variants. Arch Med Res. 2009; 40(6):428-434. [PubMed: 19853182]

6. Weaver BA. Epidemiology and natural history of genital human papillomavirus infection. J Am Osteopath Assoc. 2006; 106(3 Suppl 1):S2-8. [PubMed: 16729554] 
7. zur Hausen H. Papillomaviruses causing cancer: evasion from host-cell control in early events in carcinogenesis. J Natl Cancer Inst. 2000; 92(9):690-698. [PubMed: 10793105]

8. Cox JT. History of the use of HPV testing in cervical screening and in the management of abnormal cervical screening results. J Clin Virol. 2009; 45 1:S3-S12. [PubMed: 19651367]

9. Gerhardt CA, Pong K, Kollar LM, et al. Adolescents' knowledge of human papillomavirus and cervical dysplasia. J Pediatr Adolesc Gynecol. 2000; 13(1):15-20. [PubMed: 10742668]

10. Brown JP, Tracy JK. Lesbians and cancer: an overlooked health disparity. Cancer Causes Control. 2008; 19(10):1009-1020. [PubMed: 18551371]

11. Goodenow C, Szalacha LA, Robin LE, et al. Dimensions of sexual orientation and HIV-related risk among adolescent females: evidence from a statewide survey. Am J Public Health. 2008; 98(6): 1051-1058. [PubMed: 18445809]

12. Moszynski P. Cervical cancer virus can be transmitted through same sex relationships, report warns. BMJ. 2009; 339:b5667. [PubMed: 20042493]

13. Marrazzo JM, Koutsky LA, Kiviat NB, et al. Papanicolaou test screening and prevalence of genital human papillomavirus among women who have sex with women. Am J Public Health. 2001; 91(6):947-952. [PubMed: 11392939]

14. Diamant AL, Schuster MA, Lever J. Receipt of preventive health care services by lesbians. Am J Prev Med. 2000; 19(3):141-148. [PubMed: 11020589]

15. Lee JG, Griffin GK, Melvin CL. Tobacco use among sexual minorities in the USA, 1987 to May 2007: a systematic review. Tob Control. 2009; 18(4):275-282. [PubMed: 19208668]

16. Moscicki AB, Hills N, Shiboski S, et al. Risks for incident human papillomavirus infection and low-grade squamous intraepithelial lesion development in young females. JAMA. 2001; 285(23): 2995-3002. [PubMed: 11410098]

17. Marrazzo JM. Genital human papillomavirus infection in women who have sex with women: a concern for patients and providers. Aids Patient Care STDS. 2000; 14(8):447-451. [PubMed: 10977974]

18. Marrazzo JM, Koutsky LA, Stine KL, et al. Genital human papillomavirus infection in women who have sex with women. J Infect Dis. 1998; 178(6):1604-1609. [PubMed: 9815211]

19. Solarz, AL. Institute of Medicine (U.S.). Committee on Lesbian Health Research Priorities. Lesbian health : current assessment and directions for the future. Washington, D.C.: National Academy Press; 1999.

20. Nurses' Health Study. Brighman and Women's Hospital/Harvard Medical School. [cited 2010 August 3]; Available from: http://www.channing.harvard.edu/nhs/

21. Field AE, Camargo CA Jr, Taylor CB, et al. Overweight, weight concerns, and bulimic behaviors among girls and boys. J Am Acad Child Adolesc Psychiatry. 1999; 38(6):754-760. [PubMed: 10361795]

22. Sawaya GF. Cervical-cancer screening--new guidelines and the balance between benefits and harms. N Engl J Med. 2009; 361(26):2503-2505. [PubMed: 19940285]

23. Remafedi G, Resnick M, Blum R, et al. Demography of sexual orientation in adolescents. Pediatrics. 1992; 89(4 Pt 2):714-721. [PubMed: 1557267]

24. SAS Statistical Software. Release 9.2. Cary, NC: SAS Institue Inc.;

25. Zeger SL, Liang KY. Longitudinal data analysis for discrete and continuous outcomes. Biometrics. 1986; 42(1):121-130. [PubMed: 3719049]

26. Doty ND, Willoughby BL, Lindahl KM, et al. Sexuality related social support among lesbian, gay, and bisexual youth. J Youth Adolesc. 2010; 39(10):1134-1147. [PubMed: 20593304]

27. Ben-Natan M, Adir O. Screening for cervical cancer among Israeli lesbian women. Int Nurs Rev. 2009; 56(4):433-441. [PubMed: 19930071]

28. Walboomers JM, Jacobs MV, Manos MM, et al. Human papillomavirus is a necessary cause of invasive cervical cancer worldwide. J Pathol. 1999; 189(1):12-19. [PubMed: 10451482]

29. Fishman SJ, Anderson EH. Perception of HIV and safer sexual behaviors among lesbians. J Assoc Nurses AIDS Care. 2003; 14(6):48-55. [PubMed: 14682068] 
30. Shoveller J, Johnson J, Rosenberg M, et al. Youth's experiences with STI testing in four communities in British Columbia, Canada. Sex Transm Infect. 2009; 85(5):397-401. [PubMed: 19508967]

31. Dritz SK. Medical aspects of homosexuality. N Engl J Med. 1980; 302(8):463-464. [PubMed: 6892533]

32. Diamant AL, Schuster MA, McGuigan K, et al. Lesbians' sexual history with men: implications for taking a sexual history. Arch Intern Med. 1999; 159(22):2730-2736. [PubMed: 10597764]

33. Lindley LL, Nicholson TJ, Kerby MB, et al. HIV/STI associated risk behaviors among selfidentified lesbian, gay, bisexual, and transgender college students in the United States. AIDS Educ Prev. 2003; 15(5):413-429. [PubMed: 14626464]

34. Biro FM, Rosenthal SL, Rymarquis L, et al. Adolescent girls' understanding of Papanicolaou smear results. J Pediatr Adolesc Gynecol. 1997; 10(4):209-212. [PubMed: 9391904] 


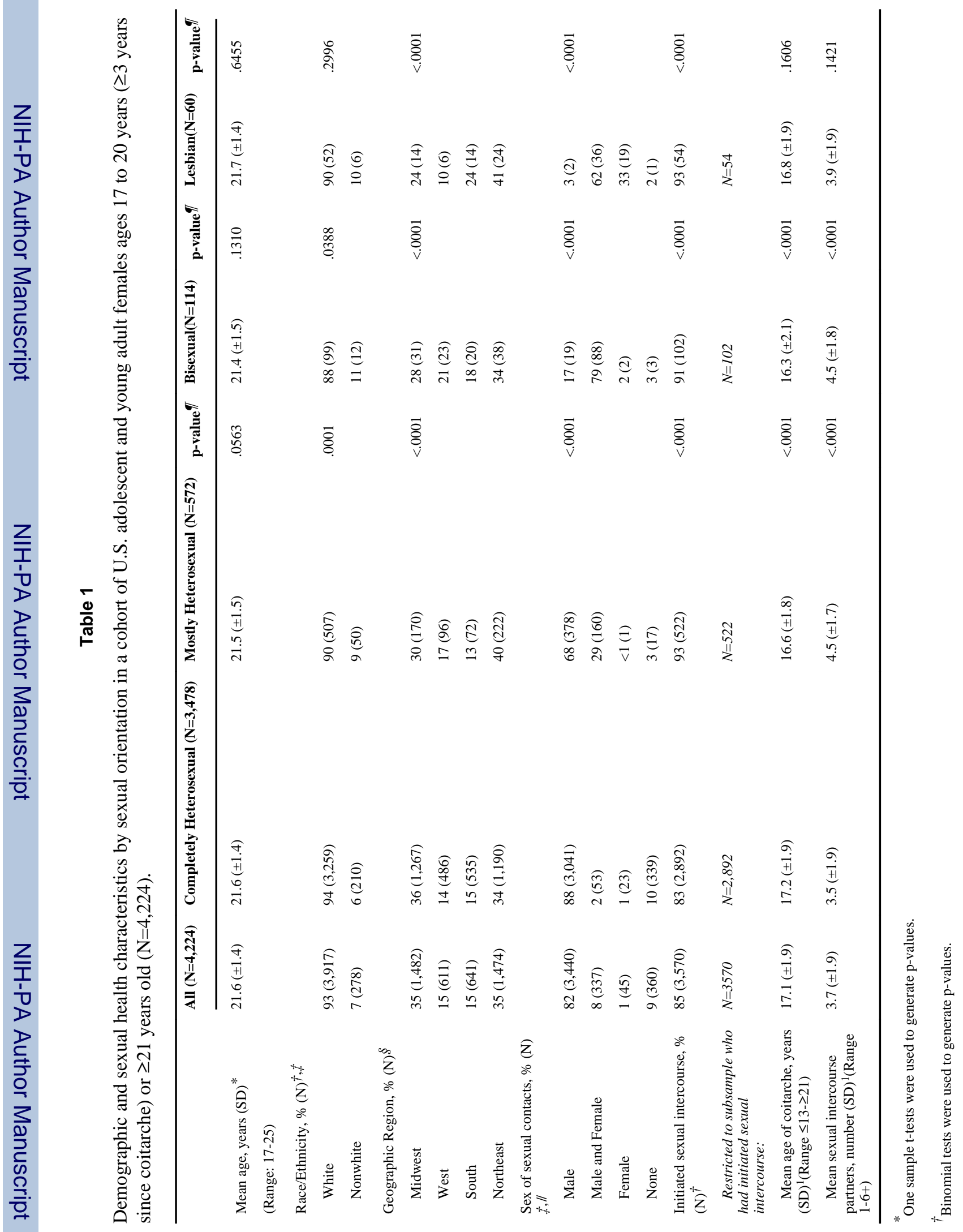




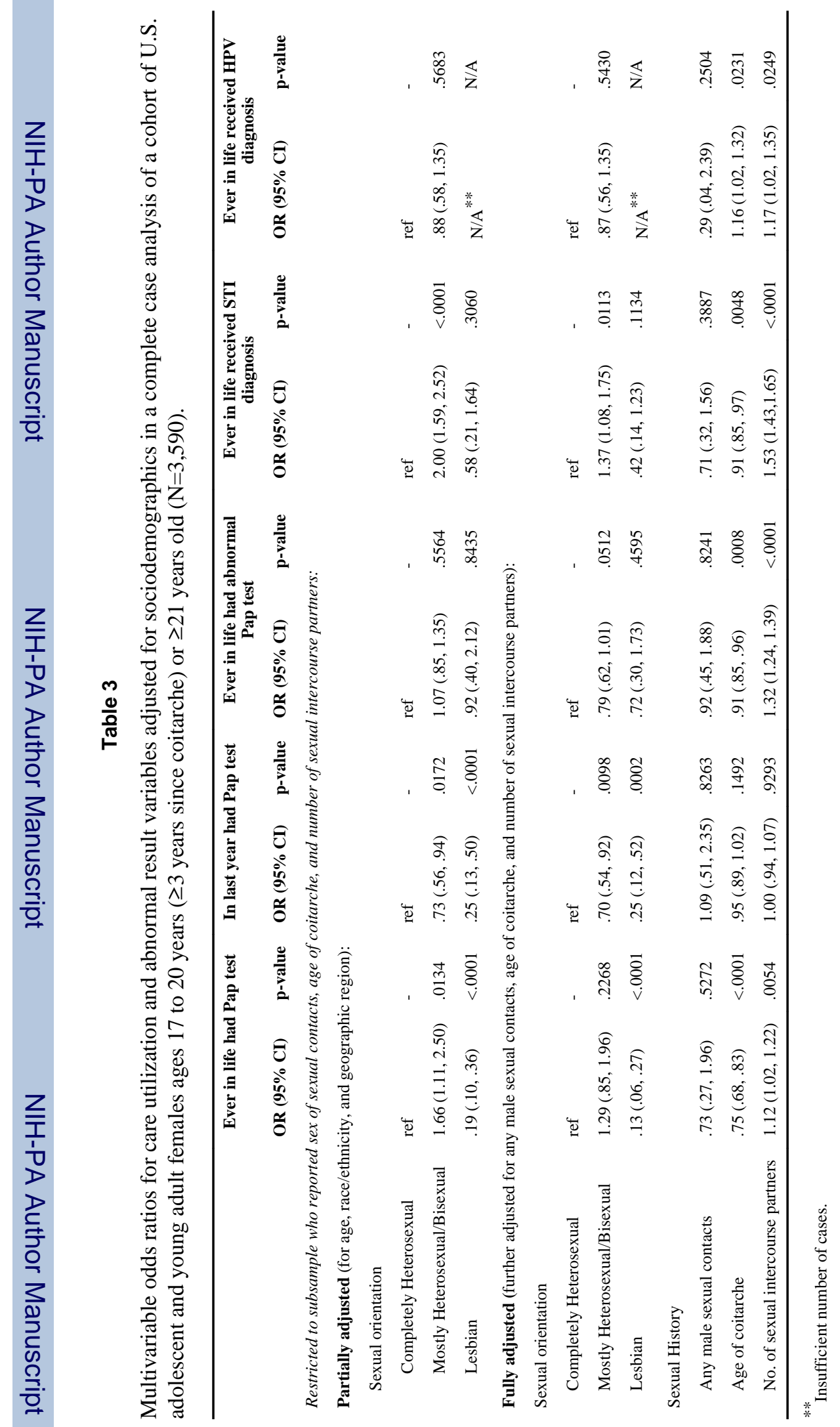

